\title{
Emulsifying Properties of Fractions Prepared from Commercial Buttermilk by Microfiltration
}

\author{
R. R. Roesch, ${ }^{1}$ A. Rincon, ${ }^{2}$ and M. Corredig ${ }^{1}$ \\ ${ }^{1}$ Department of Food Science, \\ University of Guelph, Ontario, Canada N1H 2W1 \\ ${ }^{2}$ Department of Food Science and Technology, \\ University of Georgia, Athens 30602
}

\begin{abstract}
A novel method for the separation of milk-fat globule membrane (MFGM) isolate by microfiltration in the presence of citrate was applied to prepare a fraction to be used to stabilize oil-in-water emulsions. The emulsifying properties of this fraction, containing high amounts of MFGM, were compared with a buttermilk concentrate (BMC) prepared in a similar manner but still containing the original ratio of proteins (caseins, whey proteins, and MFGM). The objective of this work was to determine if the isolation procedure would result in an ingredient with different functionality when compared with BMC. These fractions were incorporated into oil-in-water emulsions at various isolate and oil concentrations. At low concentrations of isolate, MFGM emulsions showed better creaming stability and smaller oil droplet size distribution than whole buttermilk concentrate samples. The difference in stability was attributed to the compositional difference between the 2 ingredients prepared. A selective concentration of MFGM in buttermilk by microfiltration has the potential for the development of ingredients that differ substantially from the ingredients deriving from milk or whey.
\end{abstract}

(Key words: milk-fat globule membrane, buttermilk, emulsion, casein)

Abbreviation key: BMC = buttermilk concentrate (from microfiltration), MFGM = milk-fat globule membrane.

\section{INTRODUCTION}

The aqueous phase derived from the butter making process is called buttermilk and it contains all the water-soluble material present in the milk, such as caseins, whey proteins, and lactose; it is rich in fractions

Received February 21, 2004.

Accepted August 10, 2004.

Corresponding author: M. Corredig; e-mail: mcorredi@ uoguelph.ca. deriving from the milk-fat globule membrane (MFGM). Buttermilk is considered a low value by-product of the butter industry and currently has a limited market. It has recently gained attention as a source of functional ingredients because of its low cost and the presence of MFGM (Sachdeva and Buchheim, 1997). Milk-fat globule membrane contains proteins (20 to 60\%), carbohydrates, triglycerides, and phospholipids (especially sphingomyelin, phosphatidyl choline, and phosphatidyl ethanolamine); phospholipids comprise about $33 \%$ of the MFGM (Mather, 2000). In addition to the use of MFGM as a food ingredient, it may have an important role as an active ingredient in health-related diets. Although there are no studies on the health implications of a diet rich in MFGM, several of its components have been related to health-enhancing functions (Parodi, 1997).

Milk-fat globule membrane derives from the apical cell membrane of the secretory cells of the bovine mammary gland, and it attaches to the bare fat globules when the fat globules leave the secretory cells (Keenan et al., 1993). In milk, MFGM surrounds the fat globules, stabilizing them against coalescence. For this reason it is thought that, because of their high content of phospholipids and other surface-active material, the MFGM fractions isolated from buttermilk may show good emulsifying properties. In previous work carried out isolating MFGM in the laboratory by ultracentrifugation, it was shown that, although unheated MFGM could have good functionality, the pretreatment of the cream during processing of butter affects the nature and composition of MFGM (Corredig and Dalgleish, 1998a).

Several studies have been carried out on the separation of MFGM from whey proteins, casein, and lactose present in buttermilk, but they have been limited to laboratory scale. Efforts focused on the production of MFGM fractions have been limited by the difficulty of separating the proteins deriving from skim milk. To obtain a high ratio of MFGM to skim milk proteins, especially caseins, different techniques have been used such as centrifugation (Corredig and Dalgleish, 1998a), membrane filtration (Sachdeva and Buchheim, 1997; 
Trachoo and Mistry, 1998), membrane filtration in combination with supercritical fluid extraction (Astaire et al., 2003), and separation using biosilicates (Fryksdale and Jiménez-Flores, 2001). The fractions isolated from buttermilk by ultrafiltration have been evaluated using model systems such as low fat yogurt (Trachoo and Mistry, 1998), and isolates prepared using high-speed centrifugation have been evaluated in oil-in-water emulsions (Corredig and Dalgleish, 1998a, b). Recently, more attention has been focused on microfiltration as an upscalable method to separate functional ingredients from buttermilk (Corredig et al., 2003; Morin et al., 2004).

Although MFGM may have unique functional properties compared with the other components present in skim milk, the large number of skim milk-derived proteins (especially caseins) present in buttermilk does not allow for testing of the MFGM functionality (Ramachandra Rao et al., 1995; Corredig and Dalgleish, 1998a). The removal of caseins from reconstituted buttermilk has been carried out by Sachdeva and Buchheim (1997) by precipitation of the caseins and subsequent filtration (through $0.2-\mu \mathrm{m}$ membranes) of the phospholipid extracts. Corredig and Dalgleish (1998b) separated MFGM from commercial buttermilk and demonstrated the unique properties of this material. However, the method of separation used, high-speed centrifugation, is not commercially feasible. Recently, a method was developed to produce MFGM isolates using microfiltration (Corredig et al., 2003). The development of isolation methods from buttermilk through microfiltration will increase the opportunities to produce this ingredient on a commercial scale. On the other hand, before the economics of such processes can be discussed, the differences in functionality between MFGM isolates and whole buttermilk need to be understood.

This research focused on the characterization of oilin-water emulsions prepared with MFGM isolates produced by microfiltration. These isolates were compared with a buttermilk concentrate fraction (BMC) also prepared by microfiltration. This comparative study will allow us to determine if a selective isolation of MFGM from the other components of buttermilk could result in an ingredient of unique interfacial functionality. Buttermilk concentrate fraction contains most skim milkderived proteins and MFGM in the ratio originally present in buttermilk. Although some information already exists on the emulsifying properties of MFGM extracted in the laboratory (Corredig and Dalgleish, 1998b), concentrates prepared by microfiltration have never been characterized. The results obtained in this work were also compared with the extensive literature data pres-

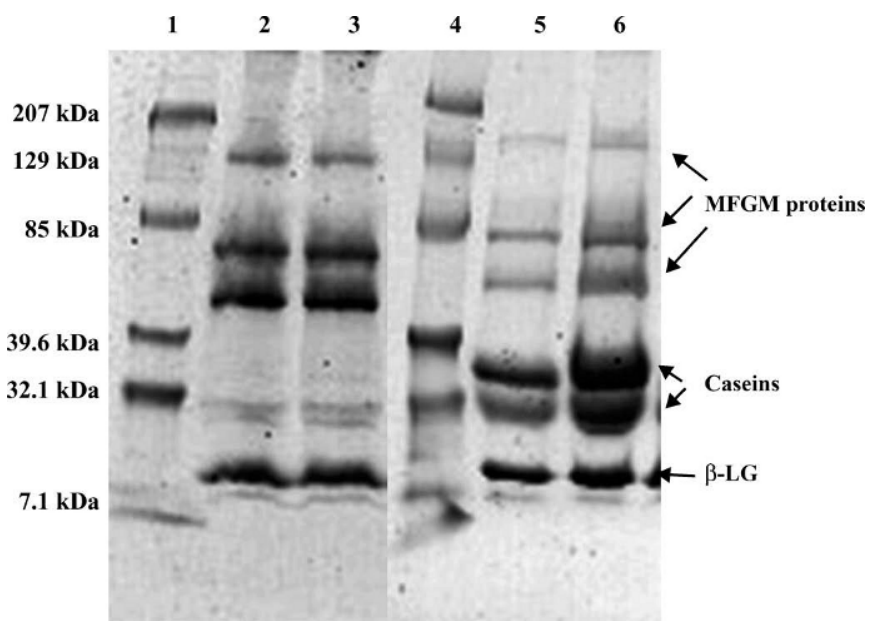

Figure 1. Sodium dodecyl sulfate-PAGE of isolates prepared using microfiltration. Lanes 1 and 4: molecular weight standard (molecular weight values are indicated on left); lanes 2 and 3: milk-fat globule membrane (MFGM) isolate; lane 5: buttermilk powder; lane 6: buttermilk concentrate (BMC). Each lane was loaded with $150 \mu \mathrm{g}$ of dried sample.

ent on the behavior of other skim milk ingredients such as caseins and whey proteins.

\section{MATERIALS AND METHODS}

Buttermilk retentate fractions were prepared from reconstituted buttermilk (Dairy Farmers of America, Kansas City, MO). Two different fractions were prepared using microfiltration as described by Corredig et al. (2003). In brief, reconstituted buttermilk (8\% solids) was filtered at $50^{\circ} \mathrm{C}$ with a pilot-scale filtration system (NIRO filtration systems, Hudson, WI) using SUPOR $100 \quad 0.1-\mu \mathrm{m}$ hydrophilic polyethersulfone membranes (Pall Gelman, Ann Arbor, MI). Retentates $(4 \times)$ were further diafiltered with water $(2 \times)$, and were then freeze-dried and stored at $-20^{\circ} \mathrm{C}$ until needed. Two fractions were prepared, a BMC and an MFGM isolate. The latter was prepared by addition of $2 \%$ sodium citrate to the reconstituted buttermilk. The presence of citrate allowed selective separation of a higher ratio of MFGM to skim milk-derived proteins (Corredig et al., 2003). The MFGM isolate contained $60 \% \mathrm{wt} / \mathrm{wt}$ protein, whereas the BMC contained $70 \%$ wt/wt protein. Figure 1 illustrates the electrophoretic migration of the proteins present in the 2 isolates prepared. Both fractions contained a higher amount of protein than the starting buttermilk powder, and although the whole buttermilk retentate showed a protein pattern similar to that of buttermilk, the MFGM isolate showed preferential concentration of the MFGM. The MFGM isolate showed a very low amount of casein and high amounts of whey proteins (mostly $\beta$-lactoglobulin). The preferential con- 
centration of $\beta$-lactoglobulin in the retentates has been previously attributed to the heat-induced protein-protein interactions occurring during the processing of butter (Corredig and Dalgleish, 1998a; Corredig et al., 2003).

Soy oil-in-water emulsions were prepared with MFGM isolate and with buttermilk retentate. Suspensions were made by dispersing using a magnetic stirrer $0.25,0.5,1$, and $2 \% \mathrm{wt} / \mathrm{wt}$ of isolate powder in $20 \mathrm{mM}$ Tris buffer, pH 7.0. Samples were stored overnight at $4^{\circ} \mathrm{C}$ to allow hydration. Emulsions were then prepared by adding soybean oil (Sigma Chemical Co., St. Louis, MO) at 3 concentrations (10, 20, and $40 \% \mathrm{wt} / \mathrm{wt})$. The samples were prehomogenized at 10,000 rpm for $1 \mathrm{~min}$ with a hand-held homogenizer (Tissue Tearor, Biospect Products, Inc., WI). Emulsions were then passed through a valve homogenizer (Emulsiflex C5, Avestin, Ottawa, Canada) at room temperature with 5 homogenization steps, the first at $5 \mathrm{MPa}$ (to ensure a homogeneous sample for the remainder of the homogenization) and the others at $70 \mathrm{MPa}$. The emulsions were stored in a cold room at $4^{\circ} \mathrm{C}$, and sodium azide $(0.02 \%)$ was added to the emulsions to prevent microbial growth. At least 2 separate emulsions were made for each treatment.

The creaming profile was measured by placing the emulsions in duplicate in graduated tubes $(10-\mathrm{mL}$ graduated tubes of $10 \mathrm{~mm}$ i.d.) and stored quiescently at $4^{\circ} \mathrm{C}$ for $20 \mathrm{~d}$. The creamed layer height was recorded as a function of time and reported as percentage relative to the total emulsion height. To determine the presence of insoluble material, fresh samples were also centrifuged at $19,000 \times g$ for $30 \mathrm{~min}$ at $25^{\circ} \mathrm{C}$.

The particle size distribution and the volume-surface average particle diameter $\mathrm{d}_{3,2}=\left(\Sigma \mathrm{n}_{\mathrm{i}} \mathrm{d}_{\mathrm{i}}{ }^{3} / \Sigma \mathrm{n}_{\mathrm{i}} \mathrm{d}_{\mathrm{i}}{ }^{2}\right)$ and $\mathrm{d}_{4,3}=$ $\left(\Sigma \mathrm{n}_{\mathrm{i}} \mathrm{d}_{\mathrm{i}}{ }^{4} / \Sigma \mathrm{n}_{\mathrm{i}} \mathrm{d}_{\mathrm{i}}{ }^{3}\right)$ of the emulsions were determined by integrated light scattering (Mastersizer S, Malvern Instruments, Southborough, MA) using the standard presentation code for samples dissolved in water (1.5295, 0.1000 , and 1.3300 for the relative particle refractive index, particle absorption, and dispersant refractive index, respectively). Measurements on emulsions containing $10 \%$ soybean oil were also carried out after incubating the samples in a solution containing $1 \%$ SDS for 20 min to break emulsion droplets aggregates.

Some of the emulsions were also analyzed with a 90 Plus particle size analyzer (Brookhaven Instruments, Holtsville, NY) to determine differences in surface $\zeta$ potential. Samples $(10 \mu \mathrm{L})$ were diluted in $10 \mathrm{~mL}$ of 1 $\mathrm{m} M \mathrm{NaNO}_{3}$ solutions at various $\mathrm{pH}$ from 2.5 to 8.0. The solutions were previously filtered through $0.22-\mu \mathrm{m}$ filters (Millex GP, Millipore Corp., Bedford, MA). The diluted solution was then transferred into cuvettes freshly rinsed with filtered buffer, and the electrophoretic mobility of the emulsion droplets was determined.

To distinguish between the types of protein adsorbed at the oil/water interface in the different samples, the cream was separated from the serum by refrigerated centrifugation of the emulsions at $20,000 \times g$ for $20 \mathrm{~min}$ at $25^{\circ} \mathrm{C}$ (Sorvall RC-5B, Fisher Scientific, Atlanta, GA). The cream phase was dispersed in $20 \mathrm{~m} M$ Tris buffer, $\mathrm{pH} 7.0$ to the initial concentration (10\% wt/wt), and centrifuged under the same conditions. Cream was dried over filter paper (Whatman no.1, Fisher Scientific). The serum was carefully removed from the centrifuge tube and filtered with a $0.22-\mu \mathrm{m}$ filter. Whole emulsions, cream, and serum were then dispersed in electrophoresis buffer [12.5\% Tris-HCl, pH 6.8, 20\% SDS (wt/ vol), $1.25 \%$ bromophenol blue, $5 \% \beta$-mercaptoethanol, and $25 \%$ glycerol] and denatured for $5 \mathrm{~min}$ in a boiling water bath. The creams and emulsions were then centrifuged at $15,000 \times g$ for 3 min with an Eppendorf centrifuge (Brinkmann Instruments, Westbury, NY) before loading. The samples were loaded onto a Criterion precast $15 \%$ Tris- $\mathrm{HCl}$ acrylamide gel (Bio-Rad, Hercules, CA). Milk-fat globule membrane and BMC fractions were also tested using the above method, but $0.015 \mathrm{~g}$ was dissolved in $1 \mathrm{~mL}$ of electrophoresis buffer, and after denaturation samples were loaded on a 4 to $20 \%$ gradient gel. Gels were run, stained with Coomassie blue, destained, and air-dried. Gels were then scanned with an imaging densitometer (GS-7000, Bio$\mathrm{Rad}$ ), and profile analysis of the intensities and position of the protein bands was carried out with imaging software (Molecular Analyst, Bio-Rad).

Photomicrographs of the emulsions were obtained with an inverted light microscope (Carl Zeiss Photomicroscope III, New York, NY) in differential interference contrast mode. The samples were carefully handled and a drop was placed on a glass slide, covered with a coverslip, and observed with a plan fluor objective. The resolution of the optical microscope was not sufficient to observe individual droplets; nevertheless, it was useful for obtaining information about qualitative differences in the floc structures.

\section{RESULTS AND DISCUSSION}

Emulsions were prepared using BMC and MFGM isolates and were then characterized for stability. Figure 2 illustrates the creaming behavior of emulsions prepared with $10 \%$ oil and MFGM or BMC. Emulsions containing $>0.25 \%$ MFGM isolate showed stability to creaming, whereas those prepared with $0.25 \%$ MFGM isolate showed cream separation within $24 \mathrm{~h}$ (Figure $2 \mathrm{~A})$. On the other hand, $10 \%$ oil-in-water emulsions containing BMC showed creaming not only when a 
A
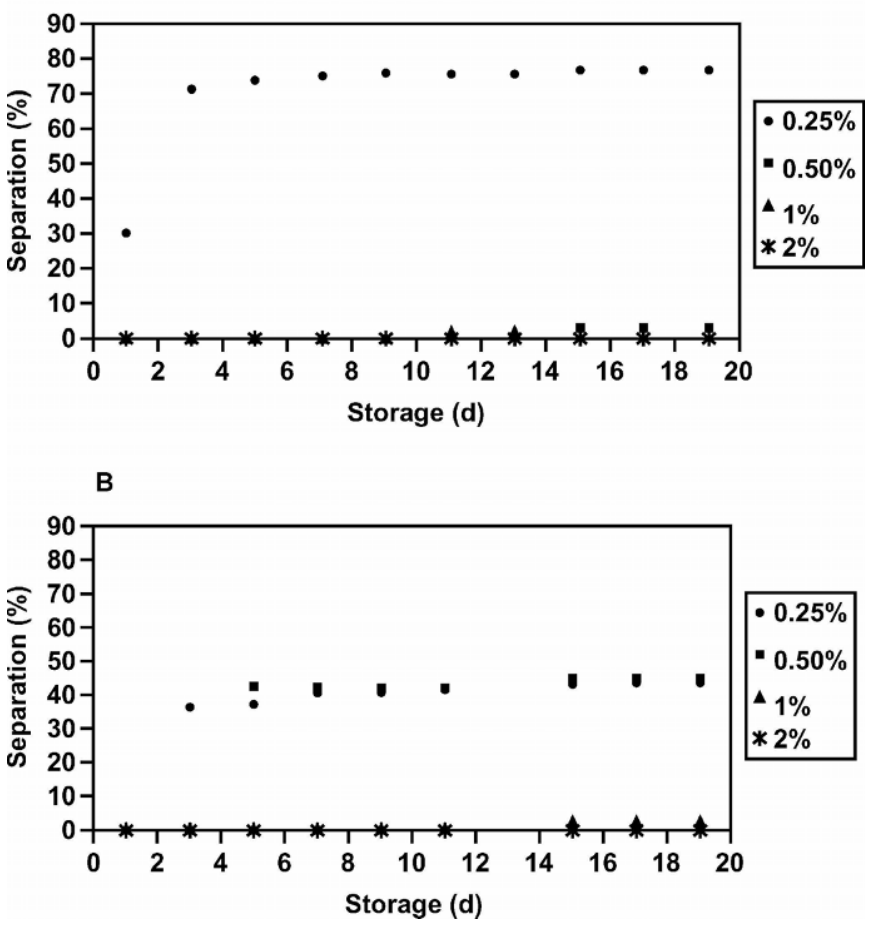

Figure 2. Stability to creaming upon quiescent storage at $4^{\circ} \mathrm{C}$ of $10 \%$ oil emulsion at various concentrations of milk-fat globule membrane (MFGM) isolate (A) and buttermilk concentrate (BMC) (B). Values are the average of at least 2 independent experiments.

$0.25 \%$ but also a $0.5 \%$ isolate was used to prepare the emulsions (Figure 2B). In samples prepared with $1 \%$ $\mathrm{BMC}$ and $10 \%$ oil, a creaming delay of $15 \mathrm{~d}$ was observed. At higher oil concentrations, all emulsions prepared with $0.25 \%$ isolates showed cream separation within $24 \mathrm{~h}$ (data not shown). At higher concentrations of isolate, there was a delay in the onset of cream separation, but all the emulsions showed some instability, even at $2 \%$ concentration of MFGM and BMC. For this reason, most of our discussion on the characterization of the 2 emulsion systems will focus on $10 \%$ oil-in-water emulsions.

The effect of homogenization at $70 \mathrm{MPa}$ on the particle size distribution of the emulsions containing 10 and $20 \%$ oil is shown in Figures 3 and 4. Emulsions prepared with MFGM isolates and $10 \%$ oil showed a monomodal size distribution with 0.5 and $0.4 \mu \mathrm{m}$ average droplet size when MFGM was used at 1 and $2 \%$ concentration, respectively (Figure 3A). On the other hand, emulsions prepared with 0.25 and $0.50 \%$ MFGM isolate showed a large population of droplets with a diameter $>15 \mu \mathrm{m}$. These results confirmed those reported for emulsions prepared with MFGM isolates using high-speed centrifugation (Corredig and Dalgleish, 1998b). In this study, whereas emulsions containing $>1 \%$ MFGM were stable
A

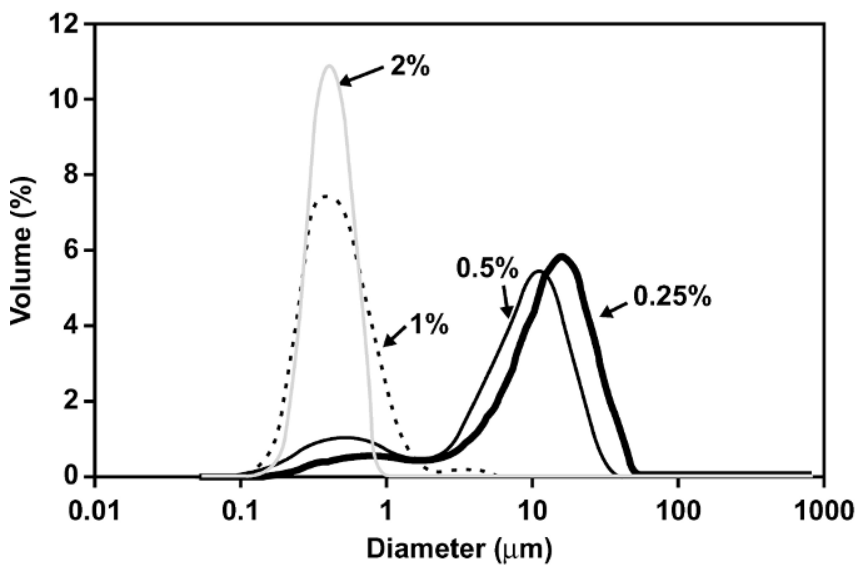

B

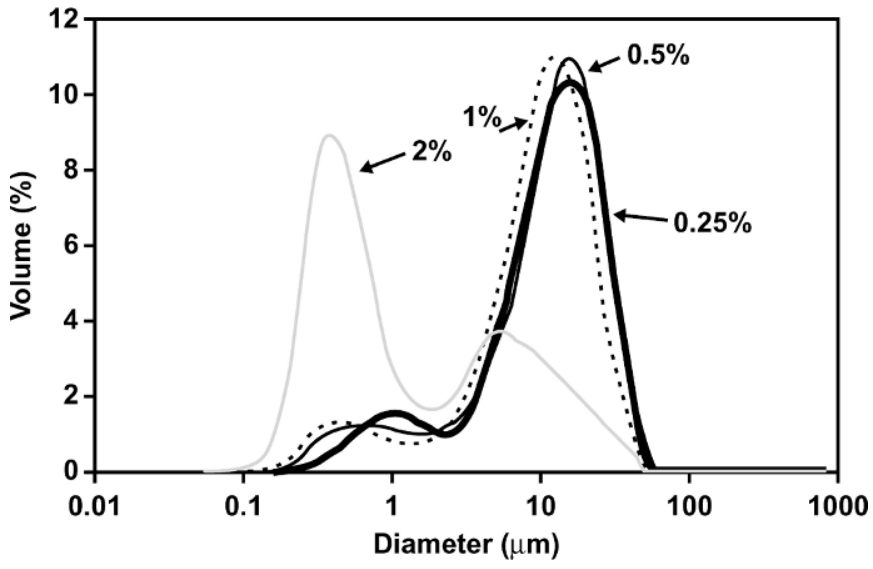

Figure 3. Particle size distribution, measured by integrated light scattering, of emulsions prepared with different concentrations $(0.25$ to $2 \%$ ) of milk-fat globule membrane (MFGM) isolate and $10 \%$ (A) or $20 \%$ (B) soybean oil. Distributions are the average of at least 2 independent experiments.

to creaming and were characterized by a monomodal size distribution, comparable emulsions containing $10 \%$ oil stabilized with BMC showed a bimodal distribution of particle sizes (Figure 4A). For example, in contrast to the monomodal distribution of a $10 \%$ oil-2\% MFGM emulsion, the emulsion containing $2 \% \mathrm{BMC}$ was characterized by one population of droplets of about $0.5 \mu \mathrm{m}$ and a second population with an average size of about $5 \mu \mathrm{m}$.

Samples containing BMC at concentrations $<2 \%$ and at all the concentrations in emulsions containing $20 \%$ oil showed a large population of aggregated particles with an average size $>20 \mu \mathrm{m}$ (Figures $3 \mathrm{~B}, 4 \mathrm{~B}$ ), regardless of the concentration of BMC used. The amount of BMC was lower than that necessary to stabilize emulsions prepared with buttermilk powder under similar conditions (Corredig and Dalgleish, 1998b), and this 


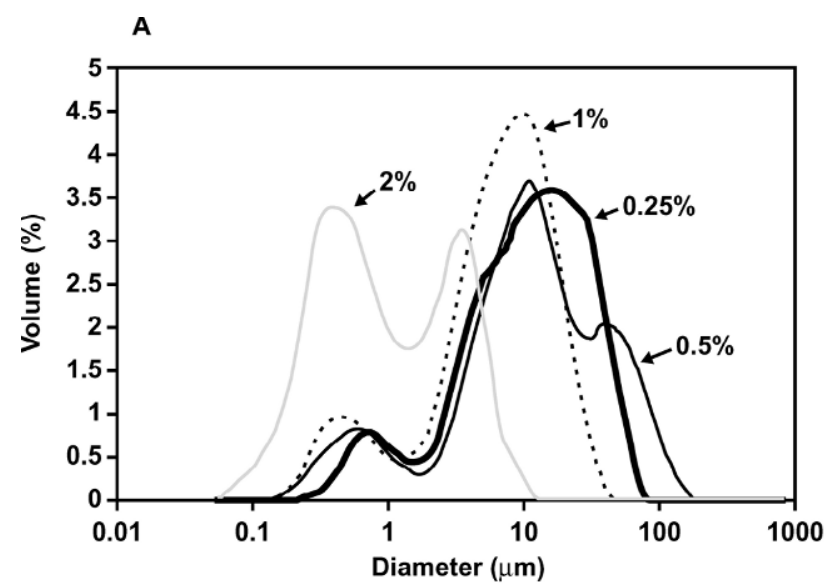

B

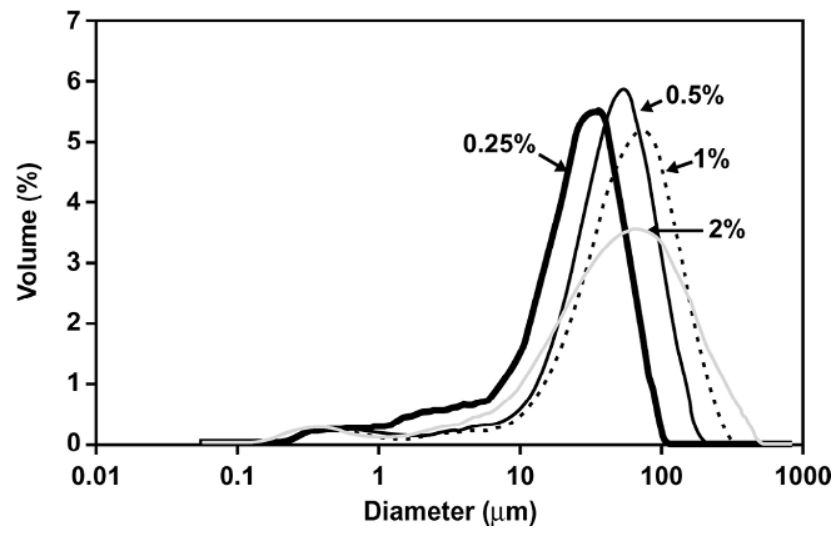

Figure 4. Particle size distribution, measured by integrated light scattering, of emulsions prepared with different concentrations $(0.25$ to $2 \%$ ) of buttermilk concentrate (BMC) and $10 \%$ (A) or $20 \%$ (B) soybean oil. Distributions are the average of at least 2 independent experiments.

may be caused by the higher protein concentration present in BMC. In emulsions prepared with BMC, casein aggregates will be in excess and will dominate the emulsifying behavior of the isolate. The more aggregated the protein, the less efficient it will be as an emulsifier. The emulsifying behavior of BMC is consistent with that recently reported for milk protein concentrates (Euston and Hirst, 1999).

It is known that during homogenization of milk and adsorption at the oil/water interface, casein micelles are disrupted and adsorb either whole or in fragments (Dalgleish, 1997). The poor emulsifying ability of micellar casein can be attributed to a concentration effect. When large aggregates are present, the effective number of protein aggregates is lower than for free dissociated caseins. In addition to less casein being available for adsorption, the association may cause the hydrophobic moieties of the proteins to be buried on the inside of the aggregates, making these systems less surface
A

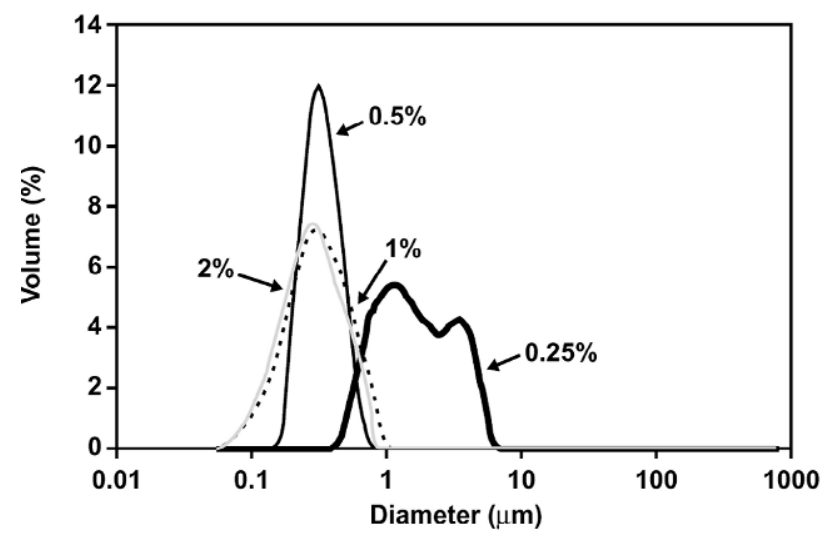

B

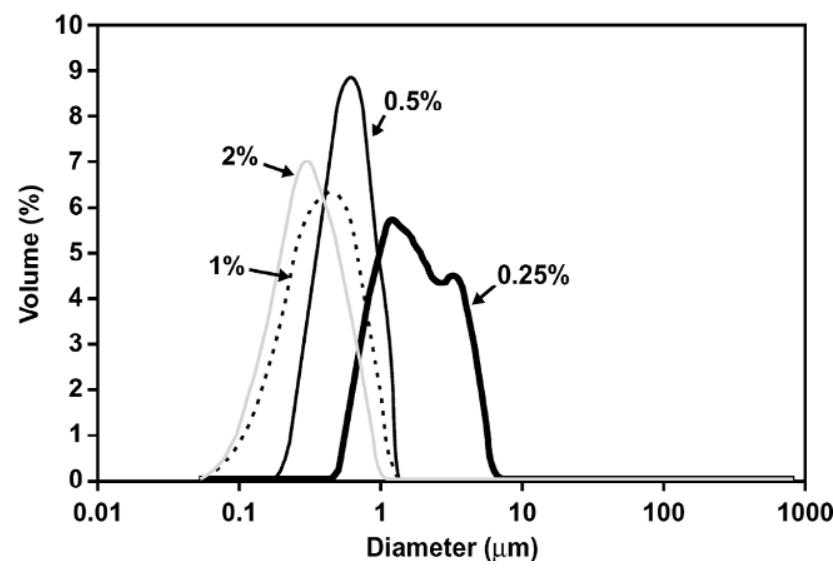

Figure 5. Particle size distribution, measured by integrated light scattering, of emulsions after incubation with $1 \%$ SDS. Emulsions were prepared with $10 \%$ soybean oil and different concentrations $(0.25$ to $2 \%)$ of milk-fat globule membrane (MFGM) isolate (A) and buttermilk concentrate (BMC) (B).

active. In our study, homogenization may have partially disrupted the casein micelles and the MFGM fragments; however, most of the isolates were still adsorbed in the aggregated form.

In emulsions prepared with MFGM isolates or BMC, creaming instability at high oil concentrations was mainly caused by adsorption of the protein isolates in an aggregated form and not enough protein present, resulting in bridging between droplets. The large particle size of these emulsions was attributed to the presence of coalesced oil droplets and bridged droplets. However, in the presence of a large amount of unadsorbed protein, phase separation due to depletion flocculation could also occur, as reported for emulsion droplets in the presence of unadsorbed aggregated caseins (Dickinson and Golding, 1997).

Incubation of SDS caused disruption of bridging flocculation with the displacement of the protein aggre- 
A

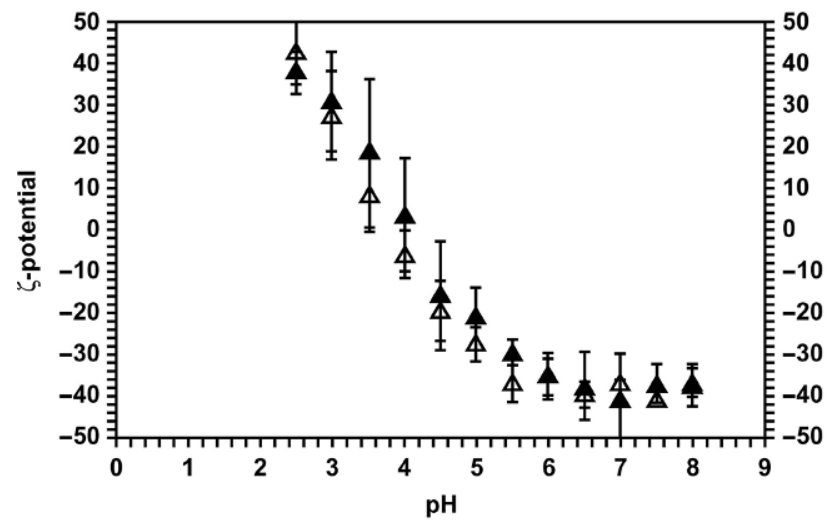

B

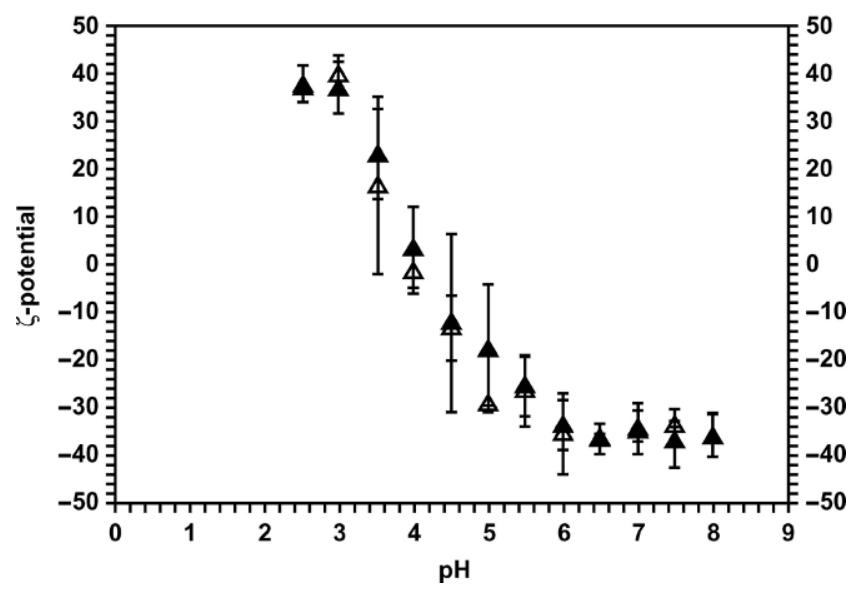

Figure 6. $\zeta$-potential vs. pH of $10 \%$ soybean oil-in-water emulsions made with $1 \%$ (A) and 2\% (B) milk-fat globule membrane (MFGM) isolate $(\triangle)$ and buttermilk concentrate $(\mathrm{BMC})(\mathbf{\Delta})$.

gates with surfactant molecules (Figure 5). By measuring the particle size in the presence of SDS, it was possible to determine the size distribution of the oil droplets and not of the aggregated flocs. When $10 \%$ oil-in-water emulsions were measured after incubation with $1 \%$ SDS (Figure 5), the oil droplet size distributions were quite different from those shown in Figures 3 and 4, indicating the presence of flocculated droplets in the emulsions prepared with $10 \%$ soybean oil in both MFGM isolates and BMC samples. In emulsions containing 0.25 and $0.5 \%$ MFGM isolate and at all concentrations of BMC, the particle size of the oil droplets was smaller after incubation with SDS, confirming that the surface of the oil droplets was only partially covered and there were bridges between droplets in close proximity.

To identify charge differences at the oil/water interface between MFGM and BMC emulsions, the effect of $\mathrm{pH}$ on the surface $\zeta$-potential of the oil droplets was determined; $10 \%$ oil-in-water emulsions stabilized by MFGM isolate were compared with those prepared with
A

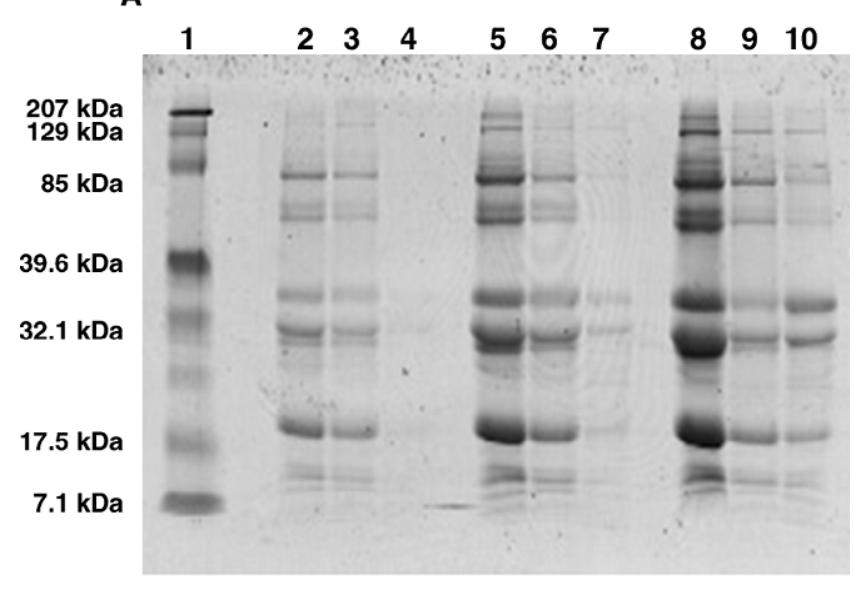

B

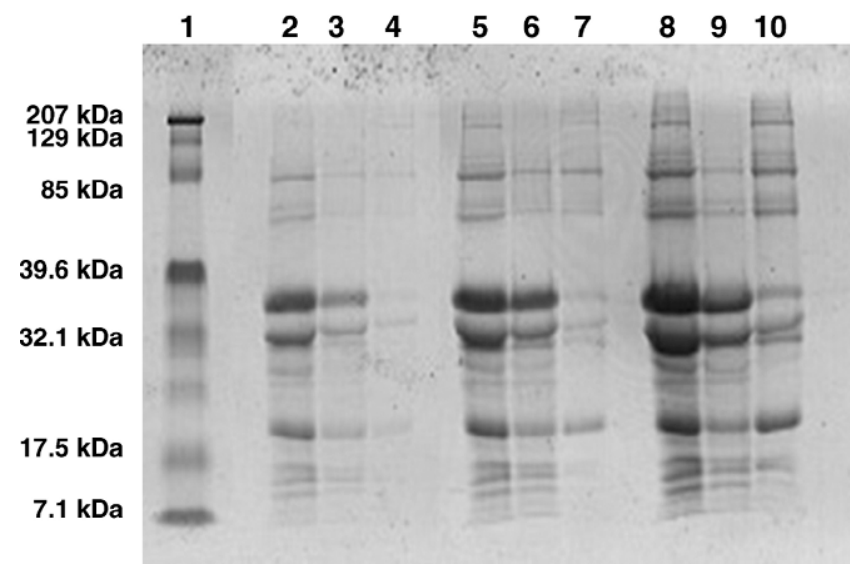

Figure 7. Sodium dodecyl sulfate-PAGE gel of $10 \%$ oil-in-water emulsions containing milk fat globule membrane (MFGM) isolate (A) and buttermilk concentrate (BMC) (B). Lane 1: molecular weight standard; lanes 2, 3, and 4: $0.5 \%$ isolate: emulsion, cream, and serum phase, respectively; lanes 5, 6, and 7: 1\% isolate: emulsion, cream, and serum phase, respectively; lanes 8, 9, and 10: $2 \%$ isolate: emulsion, cream, and serum phase, respectively.

BMC. All the emulsions showed $\mathrm{pH}$ dependence, with a decrease in surface charge with increase in $\mathrm{pH}$. Both emulsion systems showed a null droplet surface charge at a $\mathrm{pH}$ value of 3.8 to 4.0 , and the behavior did not change depending on concentration and type of protein at the interface. O'Connell and Fox (2000) previously reported similarities in $\zeta$-potential between skim milk and buttermilk. This was previously attributed to the similarity between the casein micelles; however, in this research, MFGM isolates contained high MFGM/caseins ratios.

Although no difference was shown in the surface charge behavior between emulsions containing MFGM isolate and BMC (Figure 6), the emulsions containing MFGM isolates, with a high ratio of MFGM material 
MFGM Isolate

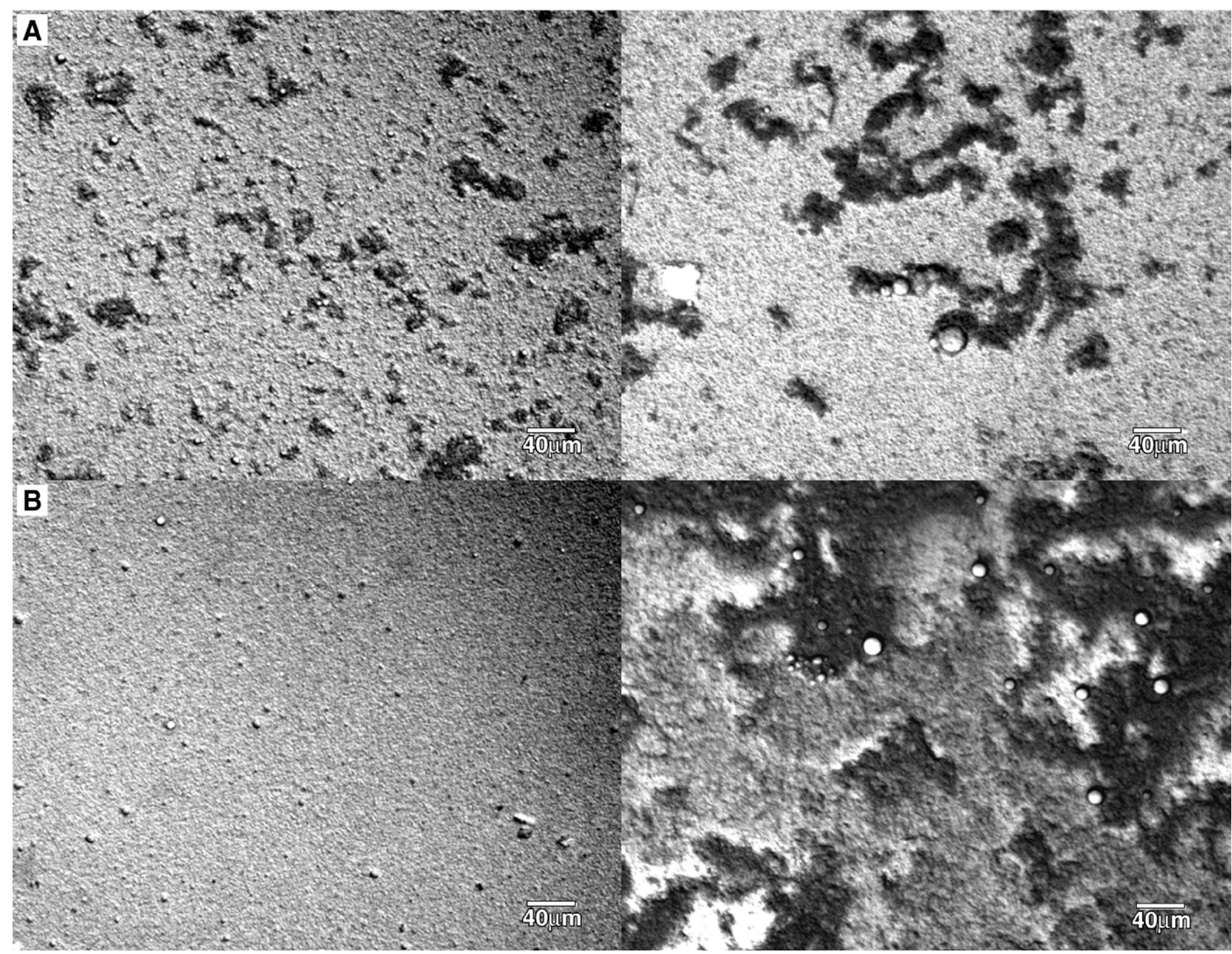

Figure 8. Images of $10 \%$ soybean oil emulsions with $0.5 \%$ (A) and $1 \%$ (B) milk-fat globule membrane (MFGM) isolate (left) and buttermilk concentrate (BMC) (right) taken by light microscopy $(225 \times)$ in differential interference contrast mode. Scale bar denotes a length of $40 \mu \mathrm{m}$.

to skim milk-derived proteins, showed better surface coverage than those containing BMC (Figures 3 and 4). This result indicated that a selective concentration of MFGM isolate from buttermilk might result in an ingredient with enhanced functionality.

The adsorption behavior of MFGM isolates and BMC in oil-in-water emulsions was studied by determining the electrophoretic pattern of the fat phase compared with that of the whole emulsion and the serum phase. Figure 7 depicts an SDS-PAGE separation of the proteins present in emulsions containing $10 \%$ soybean oil and different amounts of MFGM and BMC. Milk-fat globule membrane emulsions showed that caseins were still present in the isolate and seemed to be staining at a higher intensity than the high molecular weight proteins. Unfortunately, no data is available on the dependence of intensity of Coomassie blue staining of the MFGM proteins with concentration, and for this reason, the SDS-PAGE results were only discussed qualitatively, to determine possible preferential adsorption and distribution of the protein between the soluble and oil droplet phases.

In both emulsion systems at isolates concentrations $<0.5 \%$, analysis of the serum phase showed absence of unadsorbed protein (Lanes 2 to 4, Figure 7A and B). In other words, at these concentrations, all of the isolate was adsorbed onto the oil droplet. At higher concentrations (up to 2\%), MFGM isolates showed lower levels of unadsorbed proteins, whereas BMC emulsions showed the presence of higher amounts of caseins adsorbed and high molecular weight proteins present in the unadsorbed phase. Twenty and 40\% oil-in-water emulsions, 
regardless of the MFGM concentrations, showed patterns similar to those of $10 \%$ oil emulsions (data not shown).

Figure 8 depicts microstructural observations of emulsions prepared with $10 \%$ oil and different concentrations of MFGM isolate and buttermilk retentate. As previously mentioned, light microscopy does not allow resolution of the single oil droplets; however, it was possible to determine differences in the distribution of the flocs between emulsions prepared with MFGM and with buttermilk isolates. Examination of emulsions showed that $10 \%$ oil-in-water emulsions prepared with 0.5\% MFGM and BMC had extensive flocculation, whereas emulsions prepared with $1 \%$ MFGM isolate had individual oil droplets and no sign of flocculation. Light microscopy confirmed what was already described by integrated light scattering. In general, larger flocs were shown in BMC emulsions.

\section{CONCLUSIONS}

Emulsions containing MFGM isolates at concentrations $>0.25 \%$ and $10 \%$ oil showed small particle size distributions, with a decrease in droplet size with increasing concentration of MFGM isolate. In addition, they showed good stability to creaming. Similar emulsions prepared with BMC showed a different behavior, with extensive flocculation and, as expected, a behavior similar to that described earlier for milk protein concentrates (Euston and Hirst, 1999). Emulsion droplets prepared with MFGM isolates seemed to have a higher surface coverage than those prepared with BMC. All isolates contained a large amount of $\beta$-lactoglobulin, which was bridged to caseins and MFGM proteins as a result of the heat treatment the buttermilk underwent during processing. The instability of the emulsions was attributed to the absence of surface-active material sufficient to cover the interface and the presence of aggregated caseins and fragments of MFGM bridging the droplets.

These results indicated that a selective concentration of MFGM by microfiltration could result in an ingredient with different functional properties than those of BMC or other dairy ingredients currently available. Before the economics of the isolation process can be further discussed, more work needs to be carried out on the functional properties of the isolates obtained from buttermilk. It will be important to establish the functional value of such material. Furthermore, it is generally thought that the variability of buttermilk influences its functionality and quality, and this is the subject of ongoing studies. Recent work has demonstrated that buttermilk type influences the type of fractionation that can be obtained by microfiltration (Morin et al., 2004). However, the buttermilk used in the present study was representative of a highly heat-treated product (as shown by the presence of high amounts of $\beta$-lactoglobulin associated with caseins and MFGM), and we expect that the differences in functionality found in this research will only be amplified when the fractions are isolated from less damaged buttermilk.

\section{REFERENCES}

Astaire, J. C., R. Ward, J. B. German, and R. Jiménez-Flores. 2003. Concentration of polar MFGM lipids from buttermilk by microfiltration and supercritical fluid extraction. J. Dairy Sci. 86:2297-2307.

Corredig, M., and D. G. Dalgleish. 1998a. Characterization of the interface of an oil-in-water emulsion stabilized by milk fat globule membrane material. J. Dairy Res. 65:465-477.

Corredig, M., and D. G. Dalgleish. 1998b. Buttermilk properties in emulsions with soyabean oil as affected by fat globule membranederived proteins. J. Food Sci. 63:476-480.

Corredig, M., R. R. Roesch, and D. G. Dalgleish. 2003. Production of a novel ingredient from buttermilk. J. Dairy Sci. 86:2744-2750.

Dalgleish, D. G. 1997. Adsorption of protein and stability of emulsions. Trends Food Sci. Technol. 8:1-6.

Dickinson, E., and M. Golding. 1997. Depletion flocculation of emulsions containing unadsorbed sodium caseinate. Food Hydrocoll. 11:13-18.

Euston, S. R., and R. L. Hirst. 1999. Comparison of concentrationdependent emulsifying properties of protein products containing aggregated and non-aggregated milk protein. Int. Dairy J. 9:693-701.

Fryksdale, B. G., and R. Jiménez-Flores. 2001. Modification of buttermilk functionality with biosilicate adsorption process. Page 209 in Proc. IFT Annual Meeting, New Orleans, LA.

Keenan, T. W., D. P. Dylewski, T. A. Woodford, and R. H. Ford. 1993. Origin of milk fat globules and the nature of the milk fat globule membrane. Pages 83-118 in Developments in Dairy Chemistry. Lipids. P. F. Fox, ed. Applied Science Publisher, London, UK.

Mather, I. 2000. A review of proposed nomenclature for major proteins of the milk fat globule membrane. J. Dairy Sci. 83:203-247.

Morin, P., R. Jiménez-Flores, and Y. Pouliot. 2004. Effect of temperature and pore size on the fractionation of fresh and reconstituted buttermilk by microfiltration. J. Dairy Sci. 87:267-273.

O'Connell, J. E., and P. Fox. 2000. Heat stability of buttermilk. J. Dairy Sci. 83:1728-1732.

Parodi, P. W. 1997. Cow's milk fat components as potential anticarcinogenic agents. J. Nutr. 127:1055-1060.

Ramachandra Rao, H. G., M. J. Lewis, and A. S. Grandison. 1995. Effect of $\mathrm{pH}$ on flux during ultrafiltration of sweet whey and buttermilk. J. Dairy Res. 62:441-449.

Sachdeva, S., and W. Buchheim. 1997. Recovery of phospholipids from buttermilk using membrane processing. Kieler Milchwirtsch. Forschungsberichte 49:47-68.

Trachoo, N., and V. V. Mistry. 1998. Application of ultrafiltered sweet buttermilk and sweet buttermilk powder in the manufacture of non fat and low fat yogurt. J. Dairy Sci. 8:3163-3171. 